\title{
Characterization of microbubble generation in a confined turbulent jet
}

\author{
L. Barbaca ${ }^{1}$, P. S. Russell ${ }^{1}$, B. W. Pearce ${ }^{1}$ and P. A. Brandner ${ }^{1}$ \\ ${ }^{1}$ Cavitation Research Laboratory, Australian Maritime College, University of Tasmania, Launceston, TAS 7250, Australia
}

\begin{abstract}
Microbubble populations generated via expansion of supersaturated water through an orifice into an expansion tube, in a socalled mini-tube type device, are characterized using shadowgraphy measurements. The effect of geometric scale is investigated by testing geometrically similar devices with the orifice diameters of 0.25 and $0.5 \mathrm{~mm}$. To allow the detection of all bubbles passing through the imaged field-of-view, bubbly plume was discharged into a $0.5 \mathrm{~mm}$ thick Hele-Shaw cell, thus eliminating the need for depth-of-field correction. Saturation water was supplied at pressures between 200 and $1600 \mathrm{kPa}$. Both devices generated a poly-disperse bubble population, with dominant bubble size of about $10 \mu \mathrm{m}$. Halving the orifice diameter resulted with approximately an order of magnitude lower bubble production rate for the same supersaturated water supply pressure.

Keywords

cavitation; microbubbles; mini-tube
\end{abstract}

\section{Introduction}

Cavitation phenomena is inherently affected by the water gas content, either dissolved or free in form of microbubbles. A surface ship or a submarine typically operates in oceans and waterways that contain a naturally occurring population of bubbles, which provide a source of nuclei responsible for cavitation inception and its dynamic behaviour. The bubble concentration and size distribution vary with sea state and depth [1] and are also significantly affected by bubble generation from surface disturbances and turbulence in the wake of a vessel's hull and its appendages [2].

It has been shown that the proper scaling of both the nuclei concentration and size distribution is mandatory for model testing of some cavitation phenomena $[3,4,5]$. The nuclei concentration scales with the cube of the length scale and the concentrations of several orders of magnitude higher than those in the oceans are necessary to achieve the correct nuclei scaling in model test facilities. In order to achieve precise control of the nuclei content, a capability to artificially generate microbubble/nuclei populations with a desired size distribution is of paramount importance.

Nuclei populations of interest for cavitation modelling range over several orders of magnitude in both concentration and size. Examples of the typical nuclei populations measured at the Australian Maritime College (AMC) cavitation tunnel are shown in figure 1 . It can be seen that the naturally occurring nuclei are very sparse and generally smaller than $10 \mu \mathrm{m}$ in diameter [6]. In order to achieve a proper nuclei scaling, the tunnel water can be artificially seeded with microbubbles, however the generation and measurement of microbubble populations for all required levels of seeding remain a challenge.

Microbubble populations can be artificially generated using a variety of techniques as described by Lecoffre [7]. The technique used at the AMC for the tunnel seeding is based on socalled mini-tube type devices, producing a polydisperse microbubble population. Mini-tube type devices were first de-

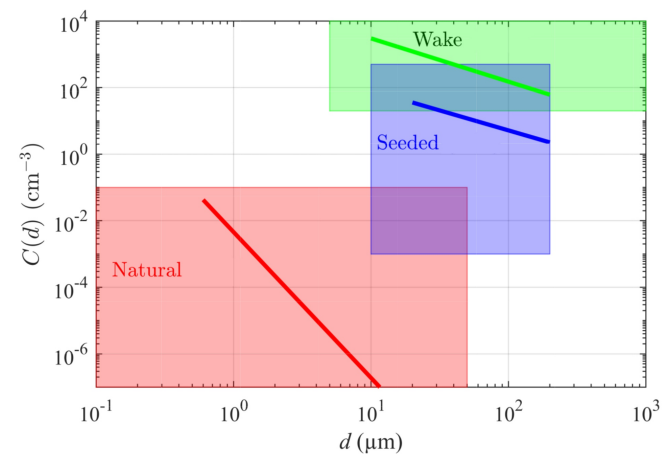

Figure 1. Nuclei distribution graph showing the bubble diameter, $d$, and concentration, $C$, ranges (shaded regions) of typical nuclei/microbubble populations of interest in cavitation experiments. The lines represent recent nuclei measurements at the AMC cavitation tunnel.

scribed in literature by Oldenziel et al. [8], and since their introduction to the AMC cavitation tunnel, mini-tubes were the subject of several studies. First measurements of the size distribution of the bubble populations generated by a mini-tube device at the AMC were reported by Brandner et al. [9]. In this study the bubbly plume was injected into a dilution flow, which was imaged using a shadowgraphy setup. In the subsequent studies, Trump et al. [10] and Giosio et al. [11] improved the measurements by discharging the diluted bubbly plume through a confined, $1 \mathrm{~mm}$ thick, radial passage, thus eliminating the need for a depth-of-field correction. In a recent study, Russell et al [12] characterized the bubble populations generated using a minitube array used for the water tunnel seeding using Mie Scattering Imaging in the tunnel test-section. A drawback of all of these measurements is a high complexity of the required experimental setups which are ill-suited to changes in the generator geometry.

Within the present study a simplified setup for the measurements of microbubble populations generated by the mini-tube devices is described. The novel setup is used to characterize the population generated by the current AMC device, which is compared with the results from the previous studies. Furthermore, a geometrically similar mini-tube device, half the scale of the original one, is tested and the effect of geometrical scaling on the size distribution of the generated bubble population is determined.

\section{Experimental setup}

The experiments were performed at the AMC Cavitation Research Laboratory using a custom designed experimental apparatus for measurement of microbubble populations. The apparatus consists of a supersaturated water vessel, a mini-tube device and a Hele-Shaw cell providing a known volume for bubble imaging.

Supersaturated water is supplied to the microbubble generator from a saturation vessel that can be pressurised up to 2000 $\mathrm{kPa}$ [9]. The saturation vessel pressure is measured using a 


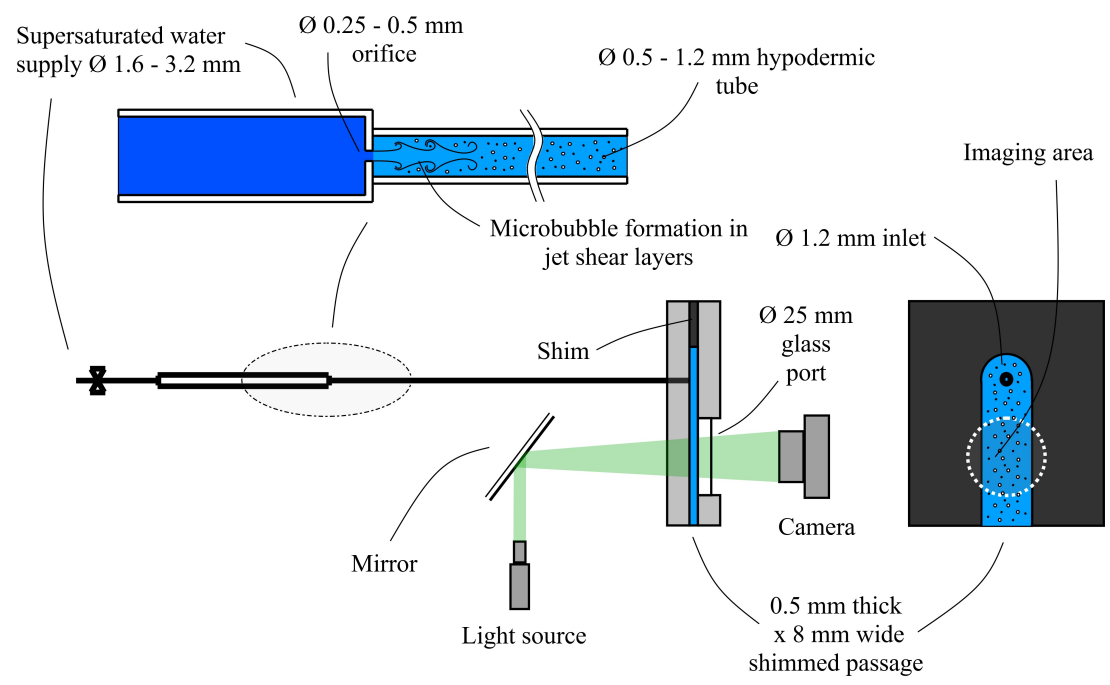

Figure 2. A schematic of the experimental apparatus used for characterization of microbubble population generated using mini-tube devices of different geometrical scale. Cross-section of the mini-tube device is presented in the upper inset. The outlet of the mini-tube device is connected to a 0.5 mm thick by $8 \mathrm{~mm}$ wide Hele-Shaw cell to eliminate the need for a depth-of-field correction. Images are obtained through a glass port mounted between the shim and an acrylic plate.

Wika gauge pressure transducer Model P-10 with a precision of $0.1 \%$. Atmospheric pressure is measured using a Vaisala Model PTB210 digital barometer with a precision of $\pm 0.03 \mathrm{kPa}$. The volumetric flow rate of supersaturated water supply to the microbubble generator is calculated based on the time rate of change of water level in an isolated pipe chamber using a Orion Instruments magnetically coupled liquid level sensor with a precision of $< \pm 0.5 \%$.

The current AMC mini-tube is a custom built device that consists of a $3.2 \mathrm{~mm}$ diameter inlet tube supplying supersaturated water through a $0.5 \mathrm{~mm}$ diameter by $0.3 \mathrm{~mm}$ thick orifice into a $1.2 \mathrm{~mm}$ diameter by $200 \mathrm{~mm}$ long expansion tube. The half-scale device is built around a Valco ZBRU21C zero-deadvolume reducing union with a $0.25 \mathrm{~mm}$ diameter bore. The inlet tube has a $1.6 \mathrm{~mm}$ diameter and the expansion tube is $0.5 \mathrm{~mm}$ diameter by $100 \mathrm{~mm}$ long. A schematic of the mini-tube device is shown in upper inset in figure 2.

A schematic of the experimental apparatus used to capture the shadowgraph images of the bubbly plume is presented in figure 2. Bubbly plume is discharged into a $0.5 \mathrm{~mm}$ thick by $8 \mathrm{~mm}$ wide Hele-Shaw cell. The cell is assembled by placing a $0.5 \mathrm{~mm}$ stainless steel shim between two transparent acrylic plates. The acrylic plate on the downstream side of the cell has a $1.2 \mathrm{~mm}$ diameter thru-hole that connects to the outlet of the expansion tube of the mini-tube device.

Shadowgraph images were obtained through a $25 \mathrm{~mm}$ diameter by $4 \mathrm{~mm}$ thick glass port using a Nikon D850 DSLR camera with a resolution of $8256 \times 5504$ pixels, equipped with a Laowa $25 \mathrm{~mm} \mathrm{f} / 2.8$ 2.5-5X Ultra Macro lens. The lens was chosen based on the manufacturer specification of $0.5 \mathrm{~mm}$ deep depth-of-field at $2.5 \mathrm{X}$ magnification, which is sufficient to capture all the bubble in the imaged field-of-view within the HeleShaw cell. The $180 \mathrm{~ns}$ pulse duration back-lightning was provided by a Sugawara Lamphouse NPL-180 controlled with a Sugawara Nano Pulse light Strobedriver NP-3A synchronized with the camera. The light was directed through the glass port by a $45^{\circ}$ Thorlabs NB1-K12 1" Nd:YaG mirror. Imaged fieldof-view was $14.4 \times 9.6 \mathrm{~mm}$, resulting with a spatial resolution of $1.74 \mu \mathrm{m} /$ pixel. The camera was positioned on a Melles Griot microstage for precise adjustment of the focal distance.
The main parameter influencing the microbubble generation from a mini-tube device is the generator cavitation number, $\sigma_{i n j}$, which can be defined as:

$$
\sigma_{i n j}=\frac{p_{d o w n}-p_{v}}{P_{u p}-p_{\text {down }}}
$$

where $p_{\text {down }}$ is the pressure at the outlet of the Hele-Shaw cell, $p_{u p}$ is the supply pressure from the saturation vessel and $p_{v}$ is the water vapour pressure. $\sigma_{i n j}$ was adjusted by varying the saturation pressure between 200 and $1600 \mathrm{kPa}$, while the outlet of the Hele-Shaw cell was open to the atmosphere. The variation of saturation vessel pressure resulted with the generator cavitation number ranging between $0.06 \leq \sigma_{i n j} \leq 0.92$. The variation in saturation vessel pressure resulted with a variation in the differential pressure between the saturation vessel and the outlet. Variation in the differential pressure affected the generator Reynolds and Weber numbers, however, the effect of change in these dimensionless parameters was shown to be less significant than the effect of change in $\sigma_{i n j}[11,12]$.

For each test condition approximately 30 shadowgraph images were acquired. A preliminary study [10] showed that, for a diluted plume, the bubble size distribution converged after processing approximately 50 images in which around 10000 bubbles were detected. The volumetric bubble concentration in the undiluted flow studied within the scope of the present study is much higher with an order of magnitude more bubble detections per image. Therefore, a lower number of images was required for the results to converge and for brevity, the detailed convergence analysis is not presented. The pressure and level data were acquired using NI LABView for 30 seconds at the sampling rate of $1 \mathrm{kHz}$.

The images were processed using LaVision Davis software package with Particle Master module. The processing was performed with a 1 pixel normalization radius, weak pixel noise reduction, $30 \%$ binarization factor, 6 pixel minimum shadow area and a $3 \%$ minimum slope. To limit the interrogation area to the part of the image with even lightning, the images were cropped, resulting with the interrogation area of $4 \times 3 \mathrm{~mm}$ and a consequent imaging volume of $6 \mathrm{~mm}^{3}$. Sample images obtained for the mini-tubes of different geometrical scale, at $\sigma_{i n j}=0.14$, 


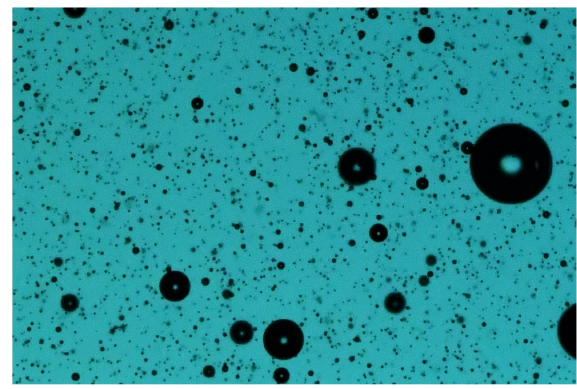

$\varnothing 0.5 \mathrm{~mm}$ orifice - mini-tube

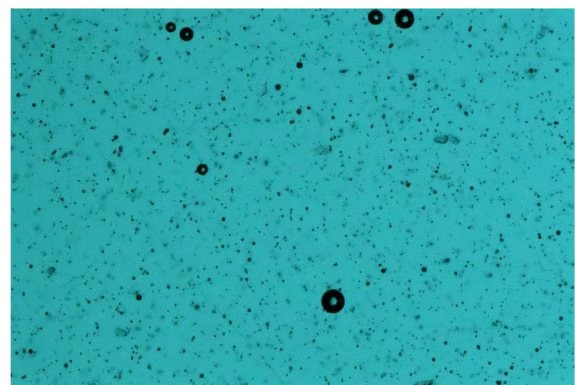

Ø $0.25 \mathrm{~mm}$ orifice - mini-tube

Figure 3. A typical example of a $1200 \times 800 \mu \mathrm{m}$ sub-sample of a shadowgraph image used to obtain microbubble size distribution and production rate.

are given in figure 3 . The detected bubbles were assigned to 2 $\mu \mathrm{m}$ bins, with the production frequency of the $i$ th bin, $f_{i}$, calculated as

$$
f_{i}=Q_{s} C_{i}
$$

where $Q_{s}$ is the measured flow rate of the bubbly plume and $C_{i}$ is the volumetric concentration of the $i$ th bin obtained from the image processing.

\section{Results and discussion}

Comparative measurements of the bubble size distribution from the mini-tube devices of different scale are presented in figure 4 in a $\log -\log$ plot. The bubble size distributions were obtained at $\sigma_{i n j}=0.14$ for both devices. It can be seen that both devices produce a polydisperse microbubble population with the dominant bubble size of about $10 \mu \mathrm{m}$. For both devices, the production frequency decreases approximately following a power law with an increase in the bubble size. A low number of detections of large bubbles (i.e. $\geq 50 \mu \mathrm{m}$ ), results with non-converged statistics and increased noise in the tail of the size distribution. While the bubble size distributions are similar for both device, it can be observed that the smaller scale device has an approximately order of magnitude lower production rate across the range of size bins presented in figure 4 . The decrease in production rate can be linked to the lower flow rate through the device stemming from a quadratic decrease in the orifice area following a decrease in the orifice diameter, however further testing is required to establish any scaling law.

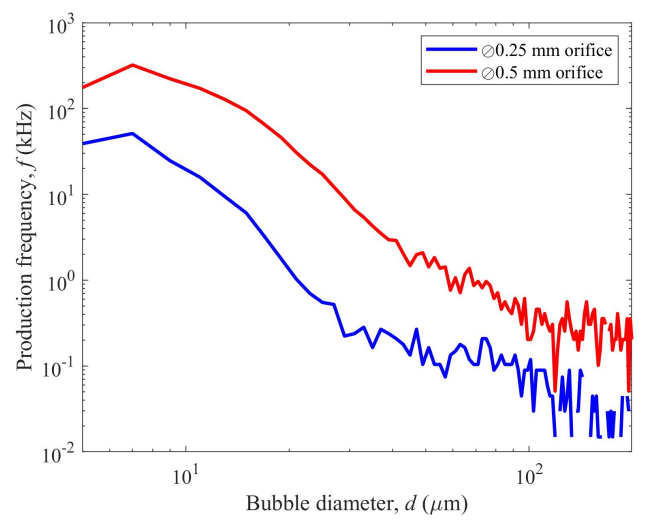

Figure 4. Comparison of the production frequency for two geometrically similar generators for $\sigma_{i n j}=0.14$. Data are presented as a histogram in a $\log -\log$ scale, with a $2 \mu \mathrm{m}$ bin width.

Bubble size distributions obtained for both devices across the range of investigated $\sigma_{i n j}$ are presented in figure 5. The maxi- mum production frequency is similar for both generators, however, it is reached at a lower $\sigma_{i n j}$ for a smaller device. Although the maximum production frequency is similar, the production of larger bubbles, i.e. with the diameter $\geq 50 \mu \mathrm{m}$, is lesser for a smaller device. The initial increase in the production frequency following a decrease in $\sigma_{i n j}$ is observed to be rather sharp for a larger device, while the smaller device exhibits a more gradual initial increase. A gradual increase in the production frequency suggests that a higher degree of the control of bubble production can be achieved with a smaller device. Additionally, the gradual increase in the bubble production rate enables a more practical (not as sensitive to the variation in saturation vessel pressure) generation of moderately dense microbubble populations.

Total production frequency summed across all the bins is presented in figure 6 for both devices for a complete range of investigated $\sigma_{i n j}$. The data points for highest $\sigma_{i n j}$ are omitted from the figure as the total bubble production rate is near zero for both devices. Again, the total production rate is significantly lesser for a smaller device at the same $\sigma_{i n j}$ value, resulting with moderately dense microbubble populations across the wider range of $\sigma_{i n j}$ values.

Total production frequency measured for the current AMC device is between 1000 and $1500 \mathrm{kHz}$, which compares favourably with the results from the previous AMC studies $[10,11]$, where total production frequencies of between 800 and $1200 \mathrm{kHz}$ were reported. The favourable comparison suggests that the novel simplified imaging apparatus is appropriate for the measurement of the bubble populations produced by the mini-tube devices.

\section{Conclusions}

A novel experimental apparatus for bubble size distribution measurement for the populations generated by a mini-tube type device was developed and successfully tested. The presented bubble size distribution and total production rate results obtained for the current AMC mini-tube device, compare favourably with the results from the previous studies, suggesting that the new apparatus can give adequate results without the complexity of the past experimental setups.

The results from the devices of different geometrical scale at the same $\sigma_{i n j}$ show that a decrease in the size of the device results with a decrease in the bubble production frequency across all the sizes. This decrease is linked to the reduced flow rate through the smaller orifice for the same differential pressure. The maximum bubble production frequency is found to be similar for both devices, however, it is achieved at a lower $\sigma_{i n j}$ for a smaller device. Moderately dense bubble populations are generated across a wider range of operating conditions for the smaller 

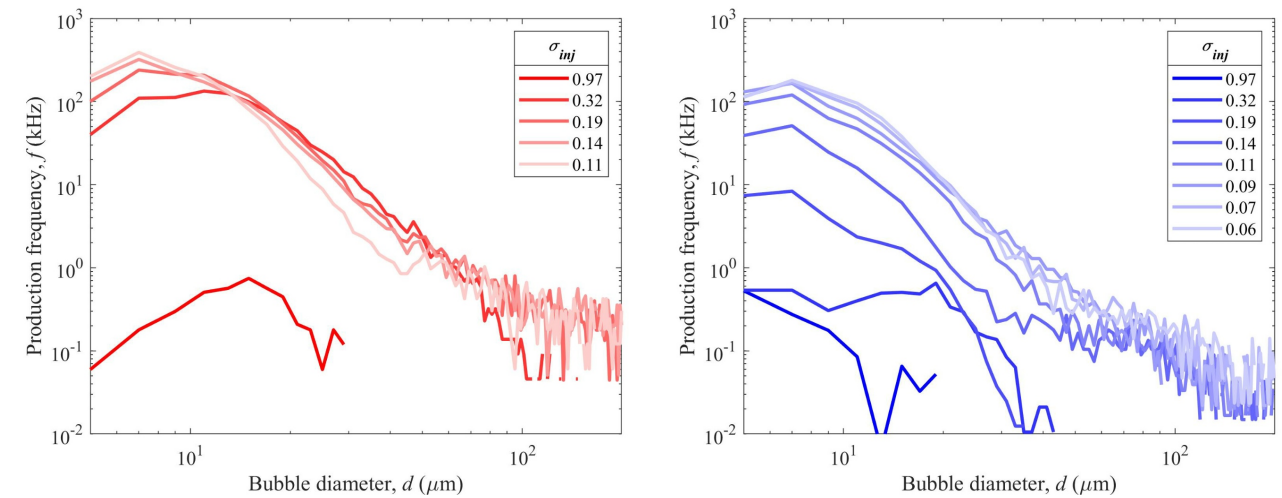

Figure 5. Production frequency histograms for $0.5 \mathrm{~mm}$ (left) and $0.25 \mathrm{~mm}$ (right) orifice diameter mini-tube devices for the range of investigated $\sigma_{i n j}$ values. Data are presented as histograms with a $2 \mu \mathrm{m}$ bin width in $\log -\log$ plots.

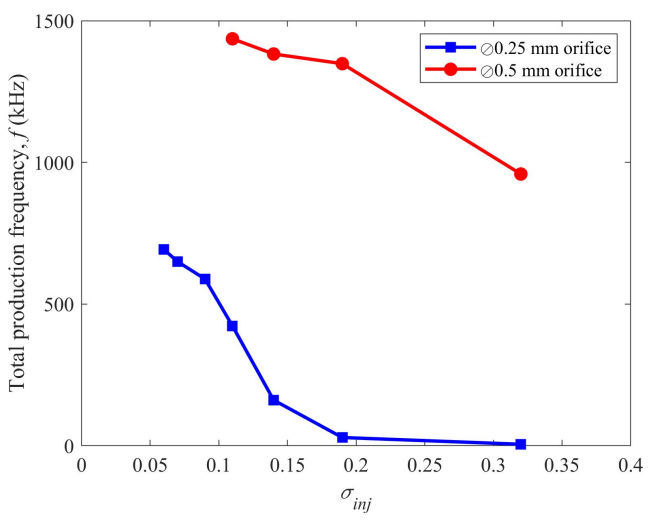

Figure 6. Comparison of the total production frequencies for the minitube devices of different geometrical scale for a range of investigated $\sigma_{i n j}$.

device, in contrast to high concentration populations generated by a large device even at high $\sigma_{i n j}$ values.

The results from the current study present a promising step in achieving a better control of the seeding in hydrodynamic facilities for moderately abundant nuclei populations. Additionally, the newly developed experimental apparatus can be used to further examine the influence of geometrical scale on the bubble populations beyond the device sizes examined in the present study.

\section{Acknowledgements}

The authors wish to acknowledge the assistance of Mr R. Wrigley and Mr S. Kent in carrying out the experiments. The authors wish to acknowledge the support of the Australian Maritime College and Defence Science and Technology Group, Australian Department of Defence.

\section{References}

[1] Deane, G. B., and Stokes, M. D. (2002). Scale dependence of bubble creation mechanisms in breaking waves. Nature, 418(6900), 839-844

[2] Castro, A. M., Li, J., Hyman, M., and Carrica, P. M. (2014). Turbulent and cavity free surface entrainment with application to ship hydrodynamics. 30th Symposium on Naval Hydrodynamics, Hobart, Australia
[3] de Graaf, K. L., Pearce, B. W., and Brandner, P. A. (2016). The influence of nucleation on cloud cavitation about a sphere. International Symposium on Transport Phenomena and Rotating Machinery - ISROMAC16

[4] Venning, J. A., Smith, S., Brandner, P. A., Giosio, D. R., Pearce, B. W. (2017). The influence of nuclei content on cloud cavitation about a hydrofoil. International Symposium on Transport Phenomena and Rotating Machinery - ISROMAC17

[5] Khoo, M. T., Venning, J. A., Pearce, B. W., and Brandner, P. A. (2018). Nucleation effects on hydrofoil tip vortex cavitation. 21st Australasian Fluid Mechanics Conference, Adelaide, Australia

[6] Khoo, M. T., Venning, J. A., Pearce, B. W., Takahashi, K., Mori, T., and Brandner, P. A. (2020). Natural nuclei population dynamics in cavitation tunnels. Experiments in Fluids, 61(2):34

[7] Lecoffre, Y. (1999). Cavitation: Bubble trackers. A. A. Balkema

[8] Oldenziel, D. M., Jansen, R. H., Keller, A. P., Lecoffre, Y., and Van Renesse, R. L. (1982). Comparison of instruments for detection of particles and bubbles in water during cavitation studies. IAHR Symposium on operating problems of pump stations and power plants, Amsterdam

[9] Brandner, P. A., Wright, G., Pearce, B., Goldsworthy, L. and Walker, G. J. (2010). An experimental investigation of microbubble generation in a confined turbulent jet. 17th Australasian Fluid Mechanics Conference

[10] Trump, M. C., de Graaf, K. L., Pearce, B. W. and Brandner, P. A. (2015). An experimental investigation of the optical measurement of microbubbles in a confined radial jet. 7th Australian Conference on Laser Diagnostics in Fluid Mechanics and Combustion, Melbourne, Australia

[11] Giosio, D. R., Pearce, B. W., and Brandner, P. A. (2016). Influence of pressure on microbubble production rate in a confined turbulent jet. 20th Australasian Fluid Mechanics Conference, Perth, Australia

[12] Russell, P. S., Barbaca, L., Venning, J. A., Pearce, B. W., and Brandner, P. A. (2020). Measurement of nuclei seeding in hydrodynamic test facilities. Experiments in Fluids, 61(79) 